\title{
Current treatment for anorexia nervosa: efficacy, safety, and adherence
}

This article was published in the following Dove Press journal:

Psychology Research and Behavior Management

15 October 2010

Number of times this article has been viewed

\author{
Lindsay P Bodell \\ Pamela K Keel \\ Department of Psychology, Florida \\ State University, Tallahassee, FL, USA
}

Correspondence: Lindsay P Bodell I 107 West Call Street, Tallahassee, FL 32306, USA

Tel + I 850645 9l4I

Email bodell@psy.fsu.edu

\begin{abstract}
Anorexia nervosa (AN) is a serious psychiatric illness associated with significant medical and psychiatric morbidity, psychosocial impairment, increased risk of death, and chronicity. Given the severity of the disorder, the establishment of safe and effective treatments is necessary. Several treatments have been tried in AN, but few favorable results have emerged. This paper reviews randomized controlled trials in $\mathrm{AN}$, and provides a synthesis of existing data regarding the efficacy, safety, and adherence associated with pharmacologic and psychological interventions. Randomized controlled trials for the treatment of AN published in peer-reviewed journals were identified by electronic and manual searches. Overall, pharmacotherapy has limited benefits in the treatment of $\mathrm{AN}$, with some promising preliminary findings associated with olanzapine, an antipsychotic agent. No single psychological intervention has demonstrated clear superiority in treating adults with AN. In adolescents with AN, the evidence base is strongest for the use of family therapy over alternative individual psychotherapies. Results highlight challenges in both treating individuals with AN and in studying the effects of those treatments, and further emphasize the importance of continued efforts to develop novel interventions. Treatment trials currently underway and areas for future research are discussed.
\end{abstract}

Keywords: anorexia nervosa, treatment, pharmacotherapy, psychotherapy, randomized controlled trials

\section{Introduction}

Anorexia nervosa (AN) is a serious psychiatric illness defined in the Diagnostic and Statistical Manual for Mental Disorders, Fourth Edition, Text Revision (DSM-IV-TR) as a refusal to maintain minimally normal body weight, an intense fear of gaining weight or becoming fat, cognitive disturbances regarding one's body weight and shape, and amenorrhea in postmenarcheal females. ${ }^{1}$ AN has been associated with one of the highest rates of mortality among all psychiatric disorders, ${ }^{2}$ due to both medical complications and suicide. ${ }^{3}$ Given the severity of AN, the establishment of both safe and effective treatments for the disorder is imperative.

Pharmacologic and psychological treatments for AN have been designed to target the core features that define AN (weight, appetite, distorted thoughts, and behaviors) as well as secondary symptoms, such as depression and anxiety. Although a variety of treatments have been tried for AN, few randomized controlled trials (RCTs) have been conducted, and few favorable results have emerged. The aim of this paper is to review RCTs in AN to summarize the efficacy, safety, and adherence associated with current treatments for AN. 


\section{Methods}

Treatment trials were identified by electronic searches of the PubMed and PsychINFO databases from 1980 to May 2010 using the search terms "anorexia nervosa", "treatment", "pharmacology", "medication", "antidepressants", "antipsychotics", "psychotherapy", "cognitive behavioral therapy", "family therapy", "inpatient", and "hospitalization". Given the current review's focus on efficacy, nonrandomized, noncontrolled treatment trials were excluded following review of abstracts and when necessary, the articles. The reference sections of all articles were reviewed to identify additional studies.

Information regarding treatment conditions, length of treatment, sample size, proportion of female participants, treatment adherence, treatment outcome, and side effects or adverse outcomes were obtained from all articles $(n=33)$ that met the inclusion criteria. Table 1 summarizes the information for pharmacotherapy trials, Table 2 for psychotherapy trials, and Table 3 for trials comparing inpatient and outpatient treatment programs. No studies compared the efficacy of pharmacotherapy to psychotherapy, and studies that examined the efficacy of medications added to psychotherapy are included in Table 1.

\section{Results Medication trials}

Several classes of medication have been examined in the treatment of AN. Evidence regarding the efficacy of pharmacotherapy for AN is limited, and there are currently no medications for the treatment of AN that are approved by the US Food and Drug Administration.

\section{Antidepressants}

The most prominently studied drug class for AN is antidepressants. Antidepressants have shown efficacy in the treatment of several Axis I disorders including major depression, obsessive compulsive disorder, and bulimia nervosa (BN). Based on shared features between $\mathrm{AN}$ and disorders that respond favorably to treatment with antidepressants, different classes of antidepressants, including tricyclics (TCAs) and selective serotonin reuptake inhibitors (SSRIs), have been evaluated in the treatment of AN.

\section{Efficacy}

Early antidepressant medication trials evaluated TCAs, including clomipramine and amitriptyline. In general, the TCAs have not proven to be effective in treating the core symptoms of AN or concurrent depression. Two RCTs evaluating the impact of clomipramine (a serotonin, norepinephrine, and dopamine blocker) as adjunctive treatment to inpatient hospitalization ${ }^{4,5}$ found no benefits of this medication on weight gain or any psychological symptoms compared with placebo, amisulpride (an antipsychotic), or fluoxetine (an SSRI). Amitriptyline, a serotonin-norepinephrine reuptake inhibitor, has also been evaluated as adjunctive treatment to inpatient hospitalization, ${ }^{6}$ as well as in an outpatient trial. ${ }^{7}$ In a five-week outpatient trial, amitriptyline was no more effective than either placebo or a no-drug comparison group in the treatment of AN or associated depressive symptoms. ${ }^{7}$ Similarly, compared with the antiserotonergic agent cyproheptadine, amitriptyline showed no improvements on weight gain or psychological symptoms beyond that of inpatient hospitalization. ${ }^{6}$ However, cyproheptadine was associated with decreased depression compared with placebo and an increased rate of weight gain and caloric intake in restricting AN patients. This benefit was offset by cyproheptadine's decreased treatment efficiency (defined as the reciprocal of days to target weight times the maximum possible days of hospitalization) in bulimic AN patients compared with amitriptyline and placebo.

SSRIs have been evaluated in both the acute and maintenance treatment phases of AN. Despite its beneficial effects in other disorders, fluoxetine does not appear efficacious in the treatment of AN in underweight ${ }^{8}$ or weight-restored ${ }^{9}$ individuals. Two RCTs have evaluated the effectiveness of fluoxetine in preventing relapse in recently weight-restored women with $\mathrm{AN}$ up to one year following hospitalization. ${ }^{9,10}$ Kaye et $\mathrm{al}^{10}$ initially found fluoxetine to be associated with improvements in weight, depression, anxiety, obsessions and compulsions, and core eating disorder (ED) symptoms from baseline to the end of the study. However, only three individuals (15\%) from the placebo group remained on treatment after one year, thus limiting comparisons between the two groups. In contrast, in a larger multisite trial of fluoxetine versus placebo in weight-restored women receiving outpatient cognitive behavioral therapy (CBT), Walsh et $\mathrm{al}^{9}$ found no differences between fluoxetine and placebo in time to relapse, percentage of patients maintaining a body mass index of at least $18.5 \mathrm{~kg} / \mathrm{m}^{2}$, or percentage of patients meeting criteria for at least a fair outcome. Additionally, there were no differences between fluoxetine and placebo on the secondary outcome measures of depression, core ED symptoms, and quality of life. However, there was a beneficial effect of fluoxetine on anxiety. Given its greater methodologic strengths, the Walsh et $\mathrm{al}^{9}$ study likely represents a more accurate 


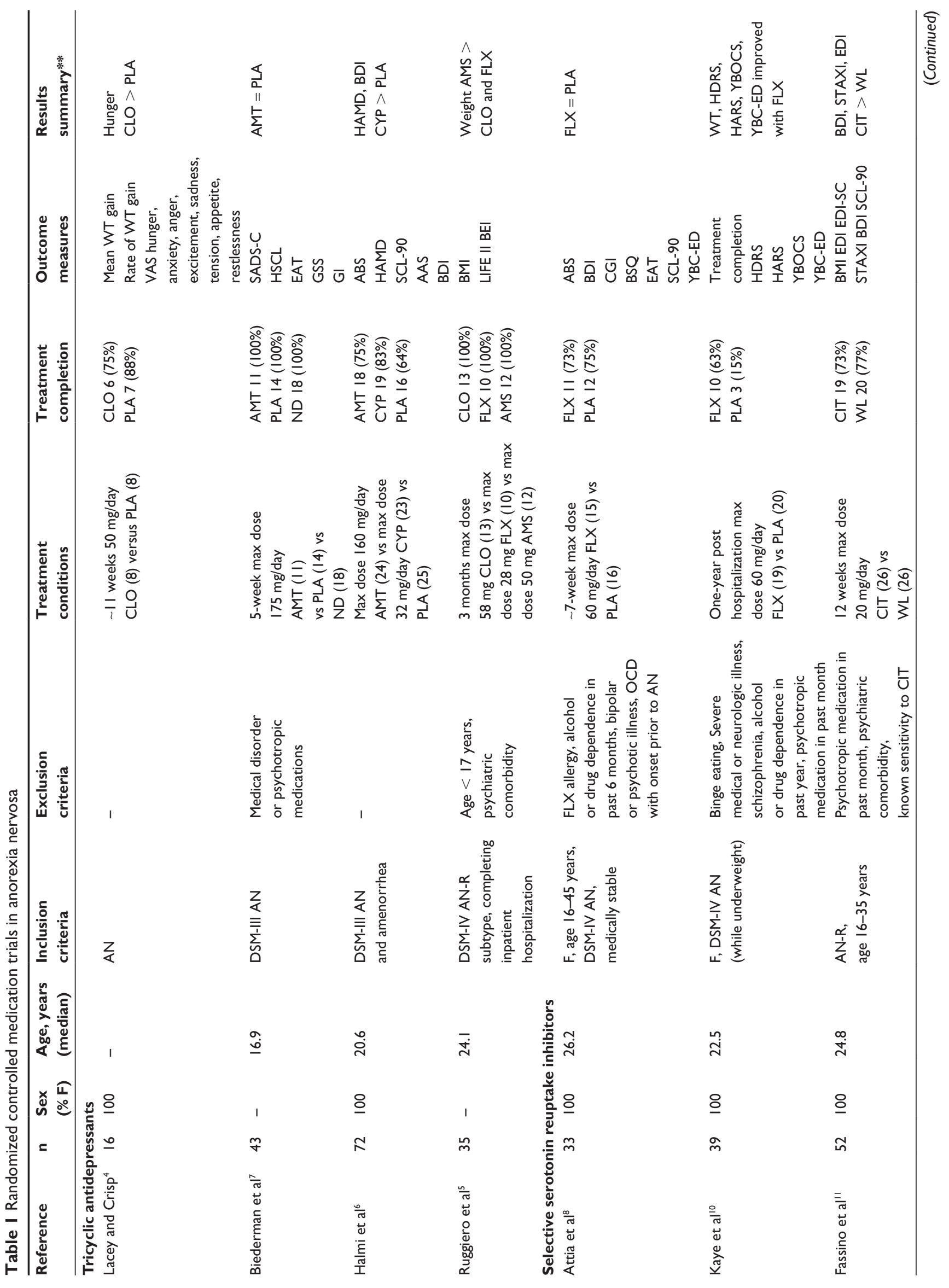




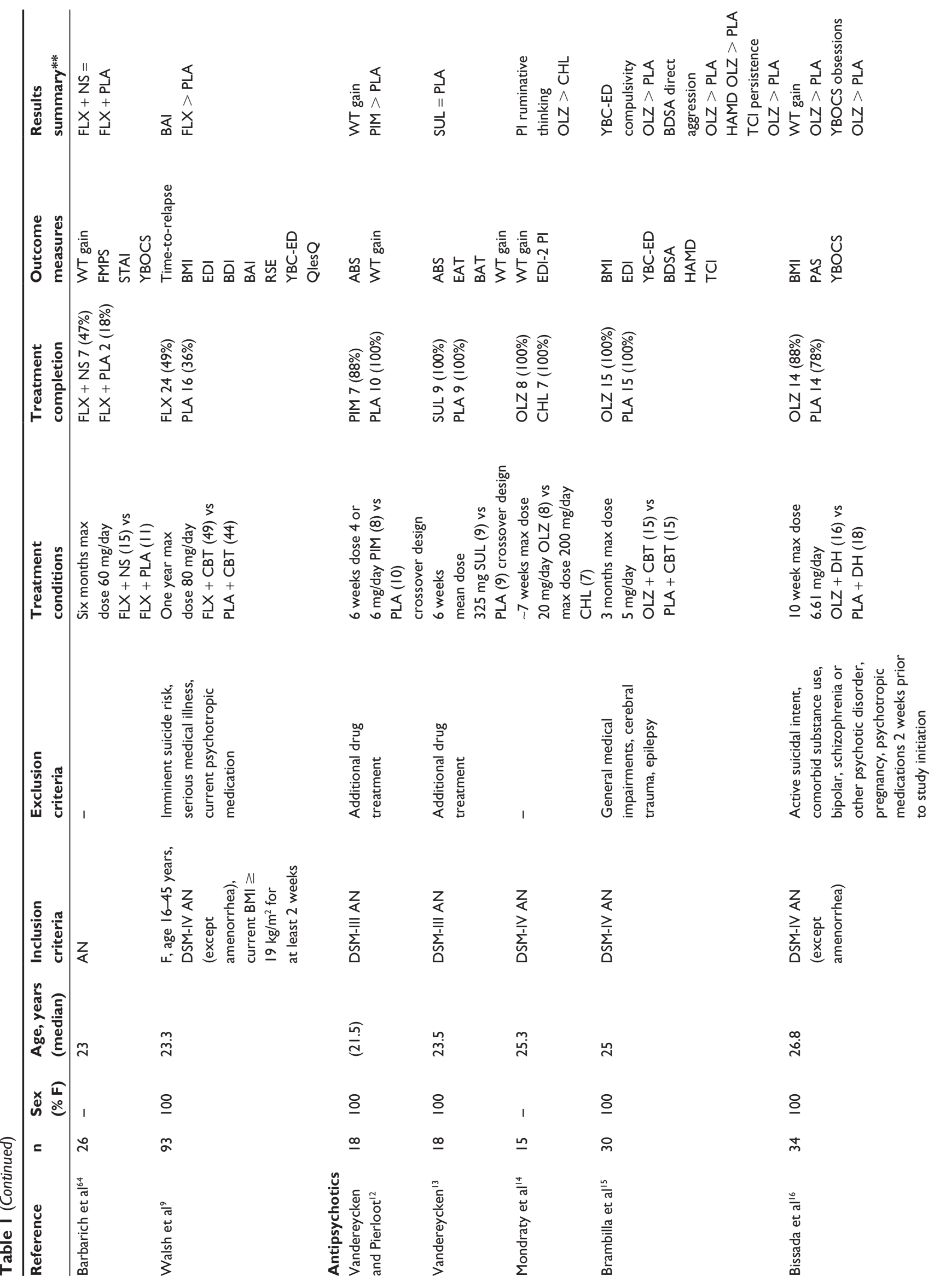




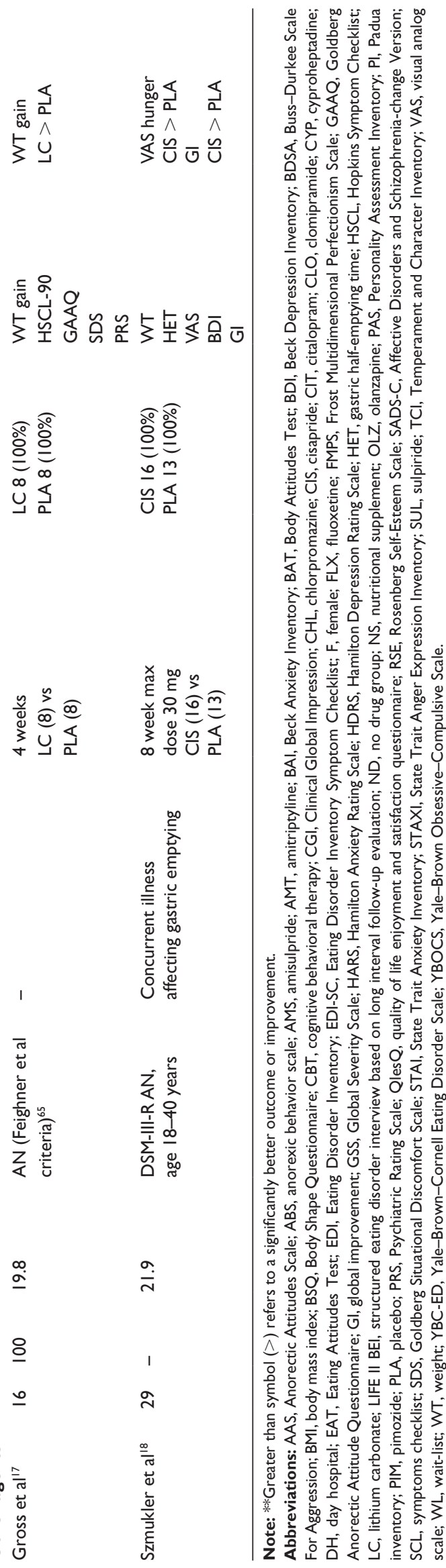

picture of lack of efficacy for fluoxetine in promoting the maintenance phase of treatment for AN.

Lastly, one RCT revealed some potential benefits of citalopram, an SSRI, in the treatment of outpatients with AN. Fassino et al ${ }^{11}$ found that compared with wait-list controls (who were monitored with periodic clinical assessments to ensure safety), citalopram was associated with improvements in depression, anxiety, and some ED symptoms; however, citalopram was not effective in increasing weight. Additionally, this study is limited by its use of a wait-list rather than a placebo control group. Thus, further evidence is needed to determine the efficacy of citalopram in the treatment of AN.

\section{Adherence}

In general, there were no differences in adherence for the TCAs compared with other medication groups (eg, placebo, amisulpride, fluoxetine, and cyproheptadine). In one study, treatment compliance for amitriptyline was good, with only two patients showing lower than expected plasma levels of the medication. ${ }^{7}$ In terms of the SSRIs, adherence to fluoxetine in hospitalized patients with AN was also good. ${ }^{8}$ There were no reported differences in treatment compliance between fluoxetine and placebo, with two patients in each group leaving treatment prematurely against medical advice. ${ }^{8}$ Treatment completion rates in the two studies examining the effect of fluoxetine in weight-restored patients with AN at one year were $63 \%{ }^{10}$ and $49 \%,{ }^{9}$ respectively. Citalopram had a relatively high rate of treatment compliance (73\%), which did not differ from placebo. ${ }^{11}$

\section{Safety}

In the early study of clomipramine versus placebo as adjunctive treatment to inpatient hospitalization, two of 16 patients, both of whom purged, complained of side effects normally attributed to TCA medications, but one patient was on placebo. ${ }^{4}$ In one study, the majority of patients treated with amitriptyline experienced substantial discomfort and adverse effects including diaphoresis, drowsiness, dry mouth, blurred vision, urinary retention, hypotension, and cardiovascular problems. ${ }^{7}$ Unlike amitriptyline, fluoxetine appears to be well tolerated by patients both in the acute and weight maintenance phases of treatment. The most common side effects or reasons for discontinuing treatment with fluoxetine during the inpatient treatment trial were persistent or worsening depression or anxiety, headaches, insomnia, and agitation. ${ }^{8}$ 


\section{Summary}

Overall, the TCA class of antidepressants appears to be ineffective in the treatment of AN, with some specific medications (ie, amitriptyline) associated with significant side effects. On the other hand, the SSRI class of antidepressants appears well tolerated by AN patients, with only mild side effects. However, SSRIs do not appear to be effective in treating symptoms of AN or preventing relapse.

\section{Antipsychotics}

Various antipsychotics have been evaluated in the treatment of AN, because affected individuals show distorted body image to a delusional proportion and because of the ego-syntonic nature of self-starvation. Furthermore, antipsychotics are known to reduce agitation and anxiety and often have weight gain as a side effect.

\section{Efficacy}

Early trials of antipsychotics have evaluated the effects of pimozide, a dopamine receptor blocker, ${ }^{12}$ and sulpiride, a selective dopamine antagonist. ${ }^{13}$ Vandereycken and Pierloot ${ }^{12}$ used a double-blind, crossover design with three-week medication periods of pimozide and placebo. Results provide modest support for enhanced weight gain using pimozide compared with placebo, but findings were limited by a small sample size $(\mathrm{n}=18)$ and a short duration of treatment (ie, three weeks of medication). Using nearly identical methods to those in the pimozide trial, Vandereycken ${ }^{13}$ found no benefits of sulpiride compared with placebo.

The most widely studied antipsychotic medication for AN is olanzapine, a thienobenzodiazepine with high affinity for serotonin and dopamine receptors. Olanzapine has been compared with both placebo and chlorpromazine (a typical antipsychotic) in RCTs. There is growing evidence to suggest that olanzapine may be beneficial in the treatment of AN, but the nature of such improvements has varied across studies. In a seven-week trial, olanzapine was more effective than chlorpromazine in reducing ruminative anorexic thinking in inpatients with $\mathrm{AN} .{ }^{14} \mathrm{In}$ a three-month outpatient trial of olanzapine versus placebo in women receiving CBT, olanzapine was associated with improvements in depression, aggressiveness, and ED-related compulsivity. ${ }^{15}$ When patients were stratified by AN subtype, there was a significant increase in body mass index for those with AN binge-purge subtype in the olanzapine group, suggesting that olanzapine may be more beneficial in increasing weight for a subgroup of those with AN. In a 10-week trial of olanzapine versus placebo in a larger sample of women in a day-hospital program, Bissada et $\mathrm{a}^{16}$ found that olanzapine was significantly better than placebo in increasing rate of weight gain and decreasing obsessions.

\section{Adherence}

Treatment adherence to pimozide and sulpiride was good, with one patient discontinuing treatment with pimozide due to side effects. ${ }^{12}$ Treatment compliance was also high in patients taking chlorpromazine $(100 \%)$ and olanzapine (88\%-100\%).

\section{Safety}

One patient in the pimozide trial reported severe central nervous system side effects and had to be withdrawn from the study. ${ }^{12}$ Furthermore, the evidence suggests that higher doses of pimozide are usually associated with central nervous system adverse effects, and these may be more pronounced in underweight individuals. ${ }^{12}$ In a small trial of chlorpromazine versus olanzapine, chlorpromazine was associated with side effects including sedation, hypotension, and blurred vision in the majority $(50 \%)$ of patients..$^{14}$ In contrast, olanzapine has been well tolerated by individuals with AN and has not been associated with reports of serious adverse effects. ${ }^{14-16}$ The most frequently reported side effect for olanzapine was mild sleepiness. ${ }^{15}$

\section{Summary}

Although antipsychotics appear to be reasonably well tolerated by individuals with $\mathrm{AN}$, some are associated with severe side effects, and those associated with the worst side effects do not appear to be associated with large improvements in treating the disorder. Of note, preliminary evidence suggests that olanzapine is well tolerated, associated with only mild side effects, and may be effective in improving some cognitive symptoms and weight.

\section{Other agents}

Other pharmacologic agents have been tried in the treatment of AN, including lithium carbonate (a mood stabilizer), cyproheptadine hydrochloride, and cisapride. Similar to some of the antipsychotic medications, lithium carbonate and cyproheptadine were evaluated in the treatment of AN because of their associations with weight gain in other populations. On the other hand, cisapride was evaluated in AN due to its known effect on increasing the rate of gastric 
emptying, which was hypothesized to decrease distress from feelings of fullness and bloating during refeeding.

\section{Efficacy}

There is some indication that lithium may facilitate weight gain in individuals with $\mathrm{AN}$, but results are limited by small sample size and need for replication. ${ }^{17}$ As previously noted, one RCT found cyproheptadine to be associated with decreased rates of depression and increased improvements in those without bulimic symptoms, but it was associated with decreased efficacy in individuals with bulimic symptoms. ${ }^{6}$ Lastly, in an eight-week trial of cisapride versus placebo, cisapride led to improvements in hunger and reductions in overall distress, but it did not prove helpful in increasing weight or ameliorating psychological symptoms. ${ }^{18}$

\section{Adherence}

Treatment compliance for lithium (100\%), cyproheptadine $(83 \%)$, and cisapride (100\%) were good.

\section{Safety}

Although there was a slight indication that lithium facilitated weight gain in a small sample, concerns have been raised that the use of lithium in individuals who may not be medically stable, and often exhibit electrolyte and fluid imbalances, may present safety issues that compromise any beneficial effects. ${ }^{19}$ Cyproheptadine was not associated with any pattern of moderately or severely rated physical symptoms or side effects in AN patients. ${ }^{6}$

\section{Psychological treatments}

A variety of psychological treatments for AN have attempted to target different aspects of the disorder, including distorted thoughts regarding food and body image, dysfunctional behaviors such as restrictive eating, binging and purging, and interpersonal and intrapsychic issues. To date, CBT has been the most frequently studied psychological treatment for AN in adults, and family therapy has gained evidence for the treatment of AN in adolescents.

\section{Cognitive behavioral therapy}

CBT for AN is based on a theoretical model that overvalued ideas about the significance of shape, weight, and control over eating are the maintaining factors of the disorder. In general, the treatment can be divided into four phases, ie, enhancement of motivation, targeting of cognitive distortions and dysfunctional behavior related to eating habits and shape or weight, issues beyond eating and weight, and relapse prevention. ${ }^{20}$ Five RCTs have been conducted comparing similar versions of CBT and cognitive therapy with other forms of psychotherapy or dietary or nutritional counseling. Four of these trials have been conducted with individuals in the acute phase of treatment, ${ }^{21-24}$ and one with weight-restored AN patients. ${ }^{25}$

\section{Efficacy}

Results from studies comparing CBT with other forms of behavioral therapy (BT) indicate that there is no difference between the two types of treatments in individuals with $\mathrm{AN}$, but these trials are limited by small sample sizes..$^{21,23}$ The first controlled trial of CBT for AN compared six months of outpatient CBT with BT and "treatment as usual" in 24 females. CBT and BT did not differ from each other in terms of improvements in weight, eating disorder symptoms, or Morgan-Russell status. ${ }^{21}$ Similarly, Ball and Mitchel123 conducted a study comparing CBT with behavioral family therapy in women aged 13-23 years. Although this study had a greater length of treatment (one year) and longer duration of follow-up (six months) than the previous study, it also found no differences between the two treatments.

Given that individuals with AN often experience a range of interpersonal problems ${ }^{26}$ and evidence that interpersonal therapy had efficacy in the treatment of BN compared with BT, ${ }^{27} \mathrm{McIn}$ tosh et $\mathrm{al}^{24}$ compared the effectiveness of 20 weeks of outpatient CBT with interpersonal psychotherapy and nonspecific supportive clinical management in a group of 56 adult women with AN. Individuals in the nonspecific supportive clinical management group showed greater global improvement than both the CBT and interpersonal therapy groups, but there were no differences between the groups in terms of weight outcome.

Lastly, CBT and cognitive therapy have both been compared with dietary and nutritional counseling in outpatients with AN. Serfaty et $\mathrm{al}^{22}$ found that weight, depression, and ED symptoms all improved with cognitive therapy in a sample of 35 adult outpatients with AN. However, all 10 individuals in the nutritional counseling control group dropped out, so no specific comparisons between the groups could be made at treatment end. Only one RCT has been conducted examining the efficacy of $\mathrm{CBT}$ in preventing relapse in individuals with $\mathrm{AN}$ compared with nutritional counseling. ${ }^{25}$ This study found that CBT was associated with significantly greater treatment success and longer time to relapse compared with nutritional counseling. Results suggest that CBT may be helpful in preventing relapse in weight-restored women with $\mathrm{AN}$ at least one year following discharge from inpatient treatment, 


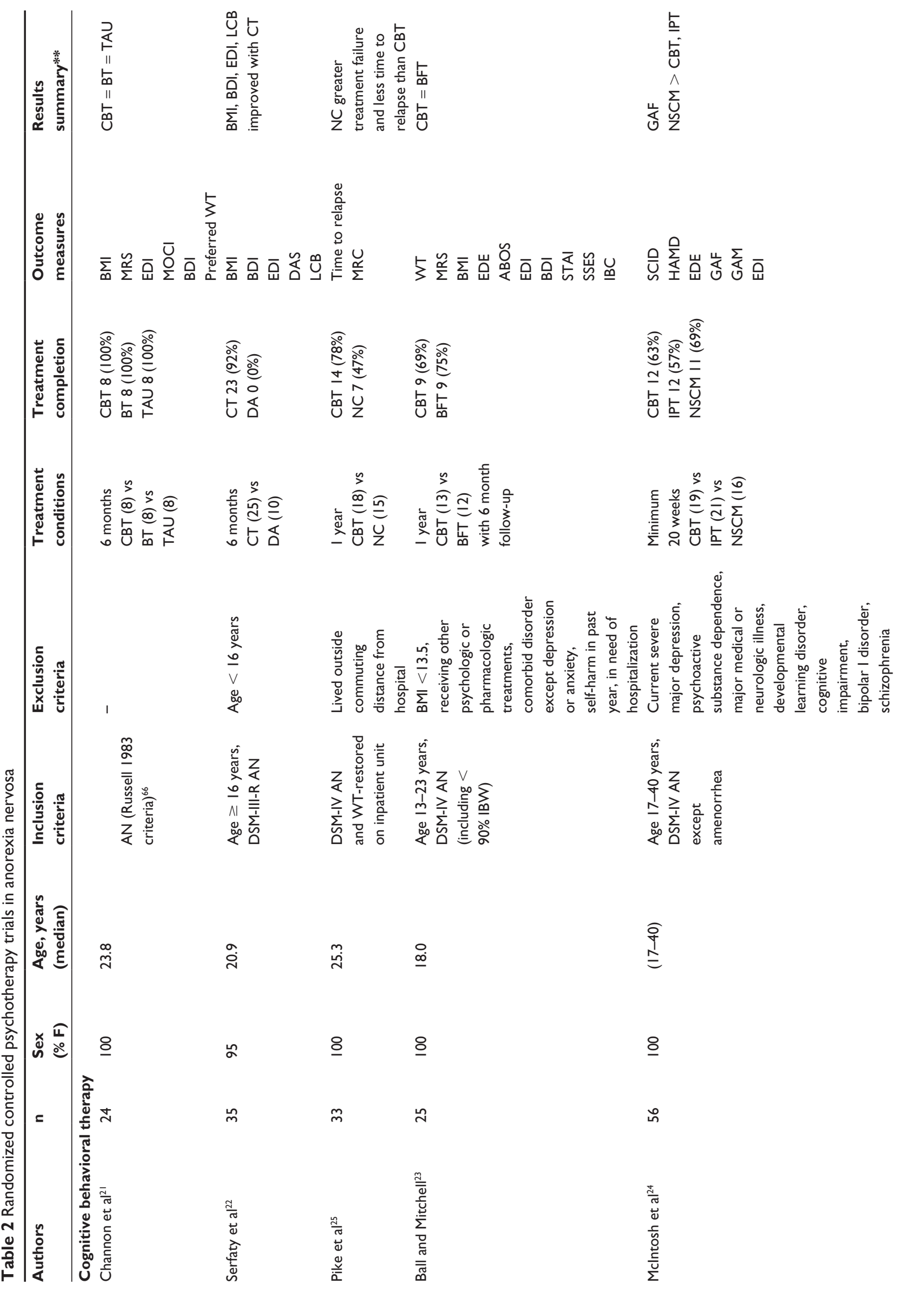




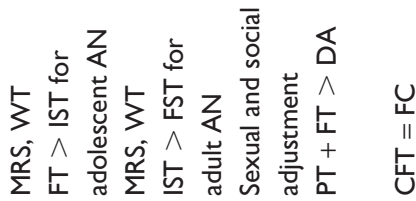

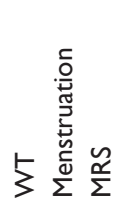

๙̊ㅇㅇ ᄚ

กิ

E는

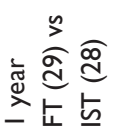

离

$+\frac{1}{x}$

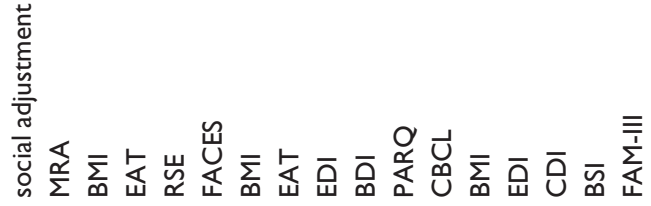

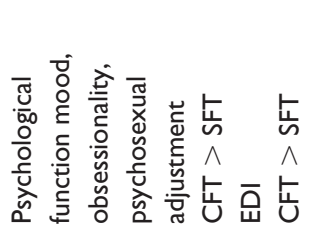

인
II
ᄂ

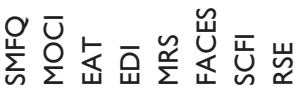

वर्य

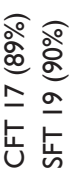

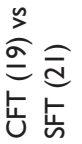

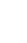
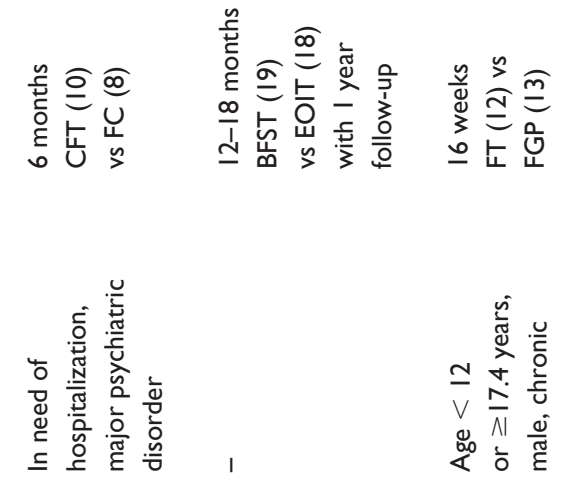

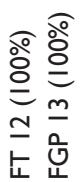

㫐梠

离焉

4

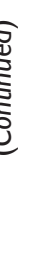

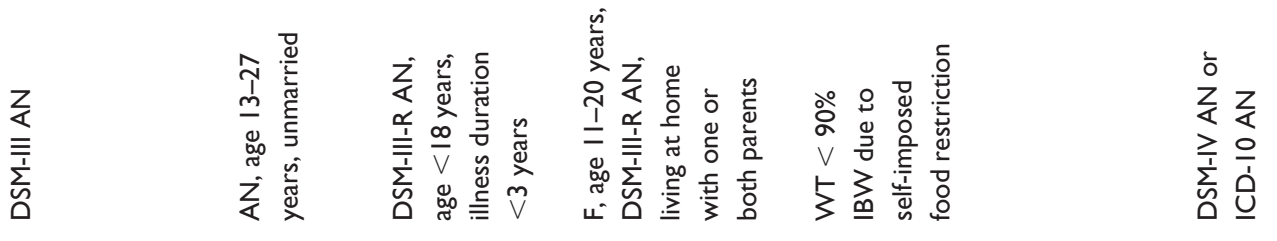

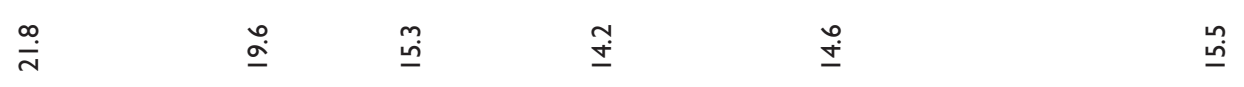

\&

으

$\infty$

으

으

$\infty$

in

$\stackrel{\infty}{\infty}$

$\hat{m}$

$\stackrel{\sim}{\sim}$

ㅇ

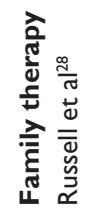

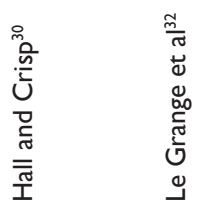

$\frac{\bar{m}}{\sqrt{0}}$
$\stackrel{0}{0}$
$\stackrel{\overline{0}}{0}$
$\stackrel{0}{\alpha}$

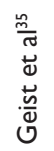

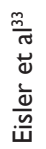




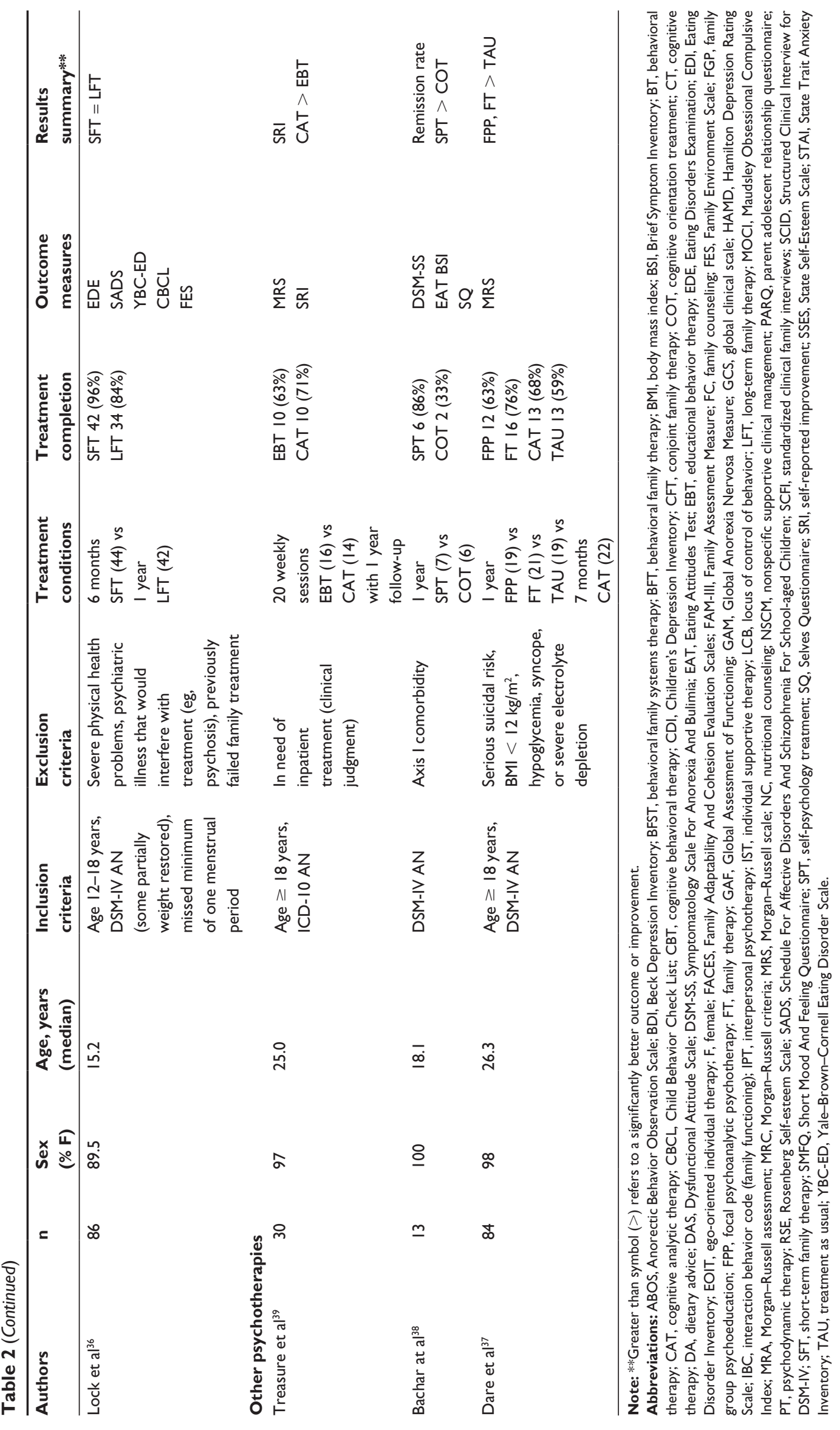


but replication is important given problems with failure to replicate positive results from other RCTs.

\section{Adherence}

Treatment completion rates for CBT in RCTs have ranged from $63 \%-100 \%$, suggesting CBT is reasonably well tolerated by patients with AN. Additionally, adherence to treatment with both CBT and behavioral therapies has been significantly better than control conditions, including nutritional counseling and dietary advice. Despite reasonable rates of treatment completion in CBT trials, no studies report compliance with homework or other aspects of the treatment, so rates of full engagement with the treatment protocol are unknown.

\section{Safety}

Although CBT is itself a "safe" treatment, some patients require hospitalization during treatment to address medical safety. In one study, three patients $(12 \%)$ had to be withdrawn from CBT or behavioral therapy and be hospitalized. ${ }^{23}$

\section{Summary}

Overall, there is some limited support for CBT improving symptoms of AN or reducing rate of relapse when compared with "nutritional" therapies, but conclusions are limited by lack of a sustained control group for one study and lack of replication for the other. Moreover, in larger trials, CBT does not seem superior to other forms of psychotherapy (eg, behavioral therapy, nonspecific supportive clinical management).

\section{Family therapy}

As previously mentioned, there is growing evidence for the efficacy of family therapy in the treatment of adolescents with AN. To date, seven RCTs have been conducted in adolescent populations, with the majority (five) being family therapy interventions. Family therapy appears superior when compared with other forms of psychotherapy, and results do not appear dependent on the format (eg, individual versus group) or length of treatment. Early theories of the development of AN assumed that dysfunctional family life was one of the causes of the disorder. As such, family therapy interpreted patients with AN as the "identified patient" and sought to treat the family system because the entire family was viewed as the actual patient. In stark contrast, the current rationale for family therapy does not blame the family for the emergence of AN, and instead is based on the idea that the family environment can be used to ameliorate the disorder. Thus, modern family therapies invite family members to become part of the treatment team to extend a therapeutic environment for change into patients' naturalistic environments.

\section{Efficacy}

Russell et $\mathrm{al}^{28}$ conducted the first RCT to examine the efficacy of family therapy. All participants were admitted to the Maudsley Hospital in London and weight restored to at least $89.5 \%$ of average body weight. Patients were first stratified into one of four subgroups and then randomly assigned to either one year of outpatient family therapy or individual supportive therapy just prior to discharge. The subgroups consisted of early-onset AN with duration less than three years (mean age 16.6 years), early-onset AN with duration greater than three years (mean age 20.6 years), AN age of onset greater than 19 years (mean age 27.7 years), and BN (mean age 24.0 years). Results indicated that family therapy was superior to individual supportive therapy for the early-onset/short duration AN subgroup, suggesting that family therapy may be beneficial for a specific subgroup of AN patients. In contrast, individual supportive therapy led to greater improvements in body weight and better Morgan-Russell outcomes ${ }^{29}$ compared with family therapy for adults with AN (ie, later-onset group). In another early outpatient trial of combined individual and family therapy versus dietary advice conducted in adults with AN, Hall and Crisp ${ }^{30}$ found that, at one-year follow-up, individuals in the combined treatment group showed significant improvements in sexual and social adjustment compared with the dietary advice treatment group; however, no differences were found for improvements in weight. In the second study of adolescent patients, body mass index was significantly more improved at one-year follow-up in adolescent girls treated with behavioral systems family therapy compared with those treated with ego-oriented individual therapy. ${ }^{31}$

Based on results indicating general benefits of family therapy in adolescents, studies have examined various permutations of family therapy, including conjoint versus separate treatment sessions, ${ }^{32-34}$ individual versus group family therapy, ${ }^{35}$ and short-term versus long-term treatment. ${ }^{36}$ Two studies comparing conjoint (ie, sessions with both the patient and parents) versus separate family therapy (ie, individual sessions for the patient and separate counseling sessions for the parents) found no differences between groups after six months ${ }^{32}$ or at five-year followup. ${ }^{34}$ Additionally, five-year follow-up revealed that 
individuals with families high in expressed emotion (maternal criticism) in the conjoint family group were less likely to experience continued weight gain after treatment, whereas those low in expressed emotion continued weight gain regardless of treatment group.

In a comparison of eight sessions of individual family versus group family psychoeducation therapy, Geist et a ${ }^{35}$ found no differences on any self-report measures of eating disorder symptoms or noneating disorder pathology. Lastly, in a relatively large family therapy trial, Lock et a ${ }^{36}$ examined the effectiveness of short (10 sessions over six months) versus long-term (20 sessions over one year) family therapy. This study found no differences between the groups for eating disorder, mood or anxiety symptoms, suggesting that the majority of adolescents with AN may benefit from short-term family therapy. However, results indicated that individuals with more severe obsessions and compulsions related to eating or those with nonintact families did significantly better in the long term compared with the short-term treatment group. Thus, identification of specific factors associated with the eating disorder may be important markers in determining the type of treatment most beneficial for a given individual.

\section{Adherence}

Adherence to family therapy in adolescents with AN has been high, with treatment completion rates ranging from $81 \%$ to $100 \%$ across studies. In the study conducted by Eisler et al ${ }^{33}$ comparing two forms of family therapy, the majority of patients remained in the study for a full year $(n=29,73 \%)$ with another seven participants completing more than three months of treatment. Adherence to family therapy has also been high in adults, with completion rates ranging from $76 \%$ to $93 \% .^{28,30,37}$

\section{Safety}

Overall, two studies reported patients needing to be admitted (or readmitted) to an inpatient program during the family therapy treatment trial. ${ }^{35,36}$ In the first study, ${ }^{35}$ five of 25 patients (20\%) receiving either individual or group family therapy needed to be readmitted to the hospital, although they all still completed the trial. Similar rates of hospitalization were found in a treatment trial comparing short-term versus longterm family therapy, with 19 of 86 patients (22\%) requiring hospitalization (average 18 days) due to medical instability. ${ }^{36}$ There were no differences in need for hospitalization between the two treatment groups, and participants re-entered their original treatment condition after hospital discharge.

\section{Other psychotherapy}

In addition to family therapy and CBT, other forms of behavioral, cognitive, and psychodynamic psychotherapies, including educational behavioral therapy (EBT), cognitive analytic therapy, cognitive orientation therapy, self-psychology, and focal psychoanalytic psychotherapy, have been studied in individuals with AN. As previously mentioned, behavioral therapies for $\mathrm{AN}$ are based on the explanatory model that dysfunctional behavioral patterns are the maintaining factors of the disorder. Thus, EBT entailed monitoring daily intake, with the overall goal of increasing the amount and variety of food consumed. In contrast, current psychodynamic therapies for AN are largely based on attachment theory and the explanatory model that dysfunctional eating patterns provide a sense of security for AN patients. Cognitive analytic therapy (CAT) follows a psychodynamic framework focusing not only on self-monitoring of behaviors but also interpersonal and transference issues. Whereas CAT combines elements of cognitive, behavioral, and psychodynamic therapies, focal psychodynamic psychotherapy focuses on the conscious and unconscious meanings of the eating disorder symptoms and how these symptoms influence current relationships. ${ }^{37}$ Selfpsychology also is a psychoanalytic therapy with the viewpoint that patients with AN cannot rely on themselves to fulfill their self needs, and thus must rely on either the avoidance or consumption of food to fulfill such needs. ${ }^{38}$

\section{Efficacy}

Treasure et $\mathrm{al}^{39}$ compared outpatient EBT with CAT in adults with AN. At one-year follow-up, results indicated that individuals in the CAT group had significantly greater self-reported improvements, but no other differences between the groups were found. Bachar et al ${ }^{38}$ reported that self-psychology was superior to cognitive orientation therapy in an RCT limited by a small sample size $(n=13)$ and high rate of drop-out $(67 \%)$ in the cognitive orientation group. Finally, to evaluate further the use of individual psychodynamic treatments and family therapy in adults with AN, Dare et $\mathrm{al}^{37}$ randomized 84 patients with AN (one male) to one of four groups, ie, one year of focal psychoanalytic psychotherapy, seven months of CAT, one year of family therapy, or one year of low-contact "routine" treatment. In terms of weight gain, those in the three treatment groups had greater improvements compared with those in the routine group. More specifically, individuals in the focal psychoanalytic or family therapy group had significantly greater weight gain compared with the routine group at 
one-year follow-up. However, despite improvements in the psychotherapy groups, more than two-thirds of the patients remained underweight at one-year follow-up. Furthermore, although the treatment groups fared better than routine treatment, it was not possible to differentiate improvements among groups.

\section{Adherence}

As previously mentioned, adolescent treatment compliance for cognitive orientation therapy was poor (33\%). Adult compliance for EBT (63\%) and CAT (71\%) was good and did not differ significantly between groups. In the study conducted by Dare et $\mathrm{al}^{37}$ patients were significantly more likely to remain in one of the psychotherapy treatment groups compared with the routine treatment group.

\section{Safety}

No patients receiving either EBT or CAT required inpatient treatment. ${ }^{39}$ In contrast, two patients $(9 \%)$ receiving CAT required hospitalization during the course of treatment. ${ }^{37}$ Hospitalization also was required for two (11\%) patients in the focal psychoanalytic group, three (14\%) in the family therapy group, and five $(26 \%)$ in the routine treatment group. ${ }^{37}$ Additionally, one patient in the routine treatment group died during the study. Overall, patients were significantly less likely to remain as an outpatient in the routine treatment compared with the three specialized treatments, suggesting improved medical safety associated with CAT, family therapy, and focal psychoanalytic therapy compared with treatment as usual. ${ }^{37}$

\section{Hospitalization and intensive multidisciplinary approaches}

Given the medical risks associated with symptoms of AN and increased risk of a fatal outcome,${ }^{3}$ inpatient and day hospitalization are widely used treatments for AN, and are often considered the surest ways to achieve weight gain in this population, given the ability to provide a controlled environment. However, only two RCTs have been conducted comparing inpatient hospitalization with other forms of treatment. ${ }^{40,41}$ Both inpatient and day programs are intensive multidisciplinary approaches generally comprised of specific behavioral refeeding programs with the end goals of medical stability and normalization of weight and eating patterns. Although hospitalization programs are often effective in normalizing weight in patients with $\mathrm{AN}$, relapse rates remain high.

\section{Efficacy}

In the first RCT of hospitalization, Crisp et $\mathrm{al}^{40}$ compared the effectiveness of inpatient treatment with two forms of outpatient psychotherapy and a dietary counseling control group in late adolescents with AN. The treatment groups comprised: inpatient treatment followed by 12 sessions of outpatient individual and family psychotherapy; outpatient individual and family psychotherapy with dietary counseling; and outpatient group psychotherapy, with groups for both the patients and parents. At one-year follow-up, individuals in the two outpatient treatment groups gained significantly more weight than those in the dietary counseling control group, but they did not differ from each other or from the inpatient group. The second RCT was conducted in a group of 167 adolescents with AN who were randomized to inpatient treatment, specialized outpatient treatment, or general child and adolescent mental health outpatient services. ${ }^{41}$ In intent-to-treat analyses at two-year follow-up, there were no significant differences between the groups in terms of Morgan-Russell outcomes, ${ }^{29}$ eating disorder symptoms, clinician-rated severity, depression, or family functioning.

\section{Adherence}

Crisp et $\mathrm{al}^{40}$ observed greater refusal to engage in treatment when randomized to inpatient treatment. Similarly, Gowers et $\mathrm{al}^{41}$ found significant variability in adherence to treatment allocation among conditions, with inpatients having the lowest rate of adherence (49.1\%) compared with both the specialized (74.5\%) and generalized outpatient (69.1\%) treatments. Although inpatient settings allow for more control over adherence to treatment protocols than outpatient settings, during voluntary hospitalization patients have the ability to leave treatment against medical advice. In general, reported drop-out rates for inpatient treatment have ranged from $20 \%$ to $50 \%$ and from $13 \%$ to $19 \%$ for day hospital treatments, ${ }^{42}$ suggesting that the latter may represent a more acceptable form of treatment.

\section{Safety}

Gowers et $\mathrm{al}^{41}$ reported that 31 of 110 individuals (28\%) assigned to the two outpatient programs required hospital admission within the year. In general, individuals with AN who are in need of hospitalization tend to be more severe and are at risk for several medical complications including gastrointestinal, endocrine, neurologic, renal, and cardiovascular problems. Although hospitalization and refeeding programs are necessary to diminish many of these life-threatening complications, in some significantly malnourished patients, 


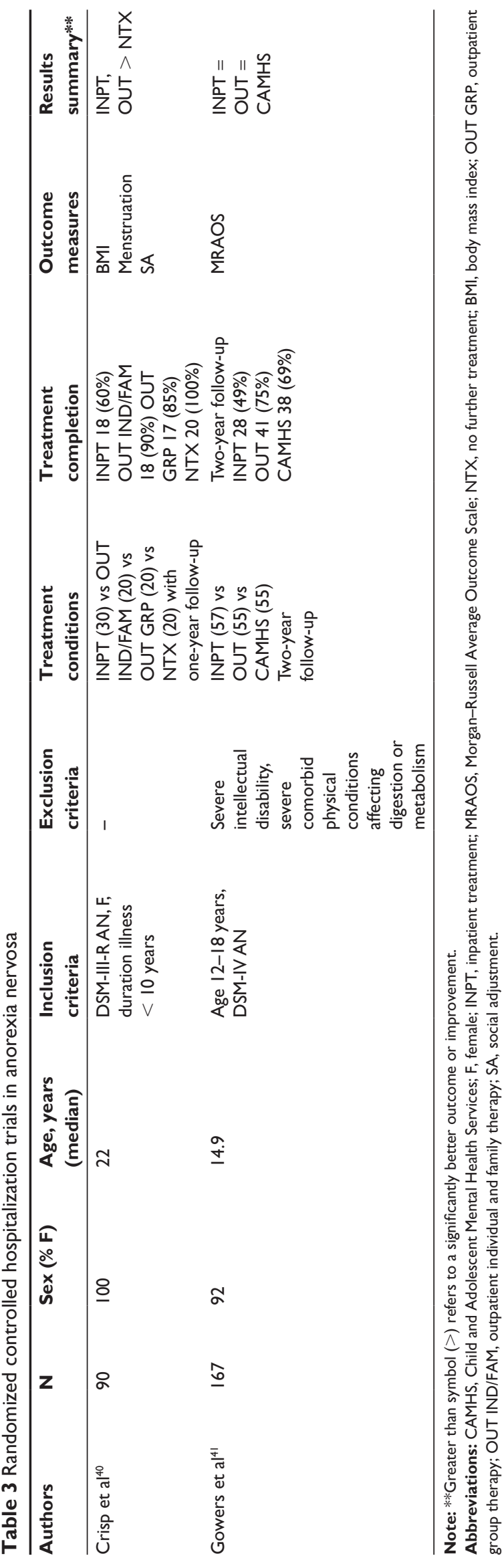

refeeding syndrome can develop in the beginning stages of nutritional rehabilitation. Refeeding syndrome is a multifaceted metabolic complication that can be fatal if not treated properly. Physician monitoring of serum potassium, phosphates, and magnesium, as well as the development of edema and vital signs is imperative, especially during early stages of refeeding. ${ }^{43}$ Neither RCT reported having patients with refeeding syndrome or severe medical complications, ${ }^{40,41}$ and no reliable estimates of this rare, but potentially fatal, complication have been provided in the literature.

\section{Discussion}

Overall, results from RCTs suggest that few treatments are adequately effective in the treatment of AN. No pharmacologic agent has emerged as a treatment of choice for AN. Unlike TCAs, SSRIs seem to be well tolerated, with low rates of adverse events or serious side effects, but there has been a failure to replicate an early finding that SSRIs might prevent relapse in weight-restored patients. Moreover, controlled trials do not support the use of antidepressants to improve weight gain, ED symptomatology, or associated psychopathology in underweight or weight-restored patients with AN. Preliminary evidence suggests that olanzapine may be beneficial in facilitating weight gain and appears to be well tolerated by patients. Thus, replication in larger samples where safety and adherence can be better assessed represents an important direction for future research.

No single psychological treatment has demonstrated clear superiority over other psychological treatments in adults with AN, not only in terms of efficacy but also in terms of patient adherence to the therapy and safety (ie, need for hospitalization). Both behavioral therapy and CBT appear effective in treating some symptoms of AN compared with nutritional counseling control conditions. However, CBT has not demonstrated greater efficacy compared with other psychological interventions. In contrast, family therapy in adolescents with AN has demonstrated efficacy compared with alternative psychotherapies in two independent RCTs, resulting in its recognition as a well established psychosocial intervention using the American Psychological Association's Task Force criteria. ${ }^{44}$ Several RCTs suggest that various forms of family therapy are effective in treating adolescents, with good rates of adherence for up to one year of treatment.

Overall, results highlight the difficulty in treating individuals with AN, and further emphasize the importance of continued efforts in developing new treatments. Thus far, treatment studies with AN have been confronted almost universally with small sample sizes due to both the low base rate of the 
disorder and its ego-syntonic nature. The disorder is relatively rare, affecting $0.5 \%$ of females in their lifetime, ${ }^{1}$ and many patients endorse lack of motivation for treatment. ${ }^{45}$

One study evaluated factors leading to nonacceptance and noncompletion in three treatment conditions (medication, CBT, and combination of medication and CBT) within a multisite $\mathrm{RCT}^{45}$ Overall, $46 \%$ of patients dropped out, with greater treatment acceptance in the CBT and combined treatment groups $(81 \%)$ compared with the medication alone treatment group (56\%). ${ }^{45}$ This finding suggests that despite any potential benefits associated with olanzapine, treatment with medication alone may not be acceptable to nearly half of patients with AN. ${ }^{45}$ Among those in the CBT or combined treatment groups, individuals with high obsessive preoccupations had greater acceptance (91\%) compared with those who had low obsessive preoccupations (52\%). Furthermore, the only predictor of treatment completion among the treatment acceptors was high self-esteem. Taken together, these findings highlight the need to improve acceptance and to reduce drop-out, especially in individuals with low self-esteem and fewer obsessive preoccupations.

In addition to low point prevalence and low motivation to engage in treatment, medical complications provoked by AN contribute to discontinuation in outpatient treatment studies. This discontinuation further contributes to small sample sizes, particularly when there are concerns about randomizing severely ill patients to an outpatient treatment condition. The severity of this disorder limits use of no treatment or placebo only comparison groups due to ethical concerns. The lack of no-treatment comparison conditions eliminates the opportunity to identify treatments as "probably efficacious" according to the American Psychological Association's Task Force criteria, and the lack of placebo comparison groups eliminates one route to developing well established treatments according to these criteria. ${ }^{46}$ Thus, when trials find no significant differences among different active treatments, it is unclear whether both treatments are effective but neither is superior or whether neither is effective.

In response to challenges faced when conducting RCTs in $\mathrm{AN}$, researchers are actively pursuing novel approaches for the treatment of AN, particularly in late adolescent and adult populations, for which no psychosocial treatment of choice has been identified. ${ }^{47} \mathrm{~A}$ recent Request for Applications from the National Institutes of Mental Health targeted the development of innovative treatments for AN, yielding funding for four treatment trials currently under way. These trials include an examination of couple-based therapy in adults, ${ }^{48}$ cognitive remediation therapy (CRT),${ }^{49}$ emotional acceptance behavior therapy, ${ }^{50}$ and food exposure therapy and ritual response prevention (AN-EX/RP). ${ }^{51}$ Each therapy is informed by interventions that have proven effective in other mental disorders, as well as an enhanced understanding of the vulnerabilities that contribute to the development of AN.

Couple-based therapy is a CBT intervention targeting core symptoms of AN as well as the unique challenges the disorder places on couples. This therapy can be conceived of as an extension of family-based therapies for children and adolescents with AN to the meaningful family unit in adults with AN. CRT is designed to address specific cognitive impairments found in $\mathrm{AN}$, and it has been found to be effective for teaching cognitive skills in other disorders (eg, schizophrenia, obsessive-compulsive disorder). In the current trial, CRT followed by CBT will be compared with CBT alone. While CRT focuses on improving cognitive skills, emotional acceptance behavior therapy emphasizes behavioral principles and strategies to attempt to increase emotional awareness and decrease avoidance of emotional negative states. Lastly, AN-EX/RP uses cognitive and behavioral techniques to address anxiety and avoidance behaviors surrounding meals specifically. In the current pilot study, six months of outpatient $\mathrm{AN}-\mathrm{EX} / \mathrm{RP}$ will be compared with $\mathrm{CBT}$ in a group of weight-restored patients with AN to examine the effectiveness of AN-EX/RP in reducing food avoidance and relapse. This treatment is based on an explanatory model in which $\mathrm{AN}$ is a fear-based disorder similar to anxiety disorders with which AN shares high levels of comorbidity ${ }^{52}$ and familial transmission. ${ }^{53}$

In addition to these four innovative treatment trials, several other treatment studies are currently under way in both adolescents and adults with AN. Using the search term "anorexia nervosa" in the World Health Organization International Clinical Trials Research Platform and the National Institutes of Health clinicaltrials.gov databases yielded 24 RCTs, with 10 having anticipated sample sizes of greater than 60 participants per treatment arm. In terms of medication, a large multisite outpatient RCT of olanzapine versus placebo is under way in adults with AN. ${ }^{54}$ Ongoing psychotherapy trials include further exploration of CBT and family therapy, as well as other forms of psychotherapy in the treatment of AN. CBT is being compared with focal psychodynamic $^{55}$ and CBT-based activity therapy ${ }^{56}$ in RCTs with sample sizes of more than 200 patients. Behavioral family therapy is being compared with individual ego-oriented therapy, ${ }^{57}$ family systems therapy, ${ }^{58}$ and family systems or individual therapy ${ }^{59}$ in adolescents, and with 
specialist supportive clinical management in adults with AN. ${ }^{60}$ A large trial with a target sample of 400 patients is comparing inpatient treatment, multifamily day treatment, and outpatient family therapy in adolescents with AN. ${ }^{61}$ Treatment setting is also being explored in a trial, comparing the effectiveness of 12-week inpatient versus day hospitalization for adolescents with AN across six hospitals. ${ }^{62}$ Lastly, one large $(n=180)$ Internet-based relapse prevention trial is testing an Internet program against treatment as usual following inpatient therapy. ${ }^{63}$ Thus, although published studies to date have been plagued by small sample sizes, there are many ongoing trials that are attempting to improve on this limitation. Given the diversity of approaches being employed, the future may provide greater insights into the efficacious treatment of AN.

In the pursuit of evidence-based treatments for AN, future research should examine moderators and mediators of treatment effects. Moderators are factors not related to the treatment condition, but identify potential subgroups for which treatment effects may be more or less powerful. For example, Lock et $\mathrm{al}^{36}$ found severity of obsessions and compulsions and nonintact families to be associated with greater improvements in the long term compared with short-term family therapy. Mediators explain how or why a given effect occurs. For example, if food exposure therapy and ritual response prevention contributes to lower anxiety around food and eating, and lower anxiety around food and eating contributes to weight gain, then the mechanism (reduced anxiety) would be understood for this intervention. Examination of both moderators and mediators can help identify which treatments may work best for whom and under what circumstances, and may in turn help increase rates of compliance and reduce rates of drop-out.

In conclusion, evidence for treatments in AN remains limited despite decades of RCTs, and there are no definitive treatments that work best for the majority of patients. For adolescent patients, family-based therapy represents a well established treatment according to the American Psychological Association's Task Force criteria ${ }^{46}$ but was not accepted as a Grade A treatment according to the National Institute for Clinical Excellence guidelines (ie, at least one RCT as part of a body of literature of overall good quality and consistency addressing the specific recommendation without extrapolation). ${ }^{47}$ Because future research is under way, it is imperative to continue to put forth effort to increase adherence and motivation for treatment in those with AN in order to conduct trials with a greater number of individuals and help patients derive all potential benefits from a given treatment.

\section{Disclosure}

The authors report no conflicts of interest in this work.

\section{References}

1. American Psychiatric Association. Diagnostic and Statistical Manual of Mental Disorders, Fourth Edition, Text Revision. Washington, DC: American Psychiatric Association; 2000.

2. Harris EC, Barraclough B. Excess mortality of mental disorder. Br J Psychiatry. 1998;173:11-53.

3. Keel PK, Dorer DJ, Eddy KT, Franko D, Charatan DL, Herzog DB. Predictors of mortality in eating disorders. Arch Gen Psychiatry. 2003; 60(2):179-183.

4. Lacey JH, Crisp AH. Hunger, food intake and weight: The impact of clomipramine on a refeeding anorexia nervosa population. Postgrad Med J. 1980;56 Suppl 1:79-85.

5. Ruggiero GM, Laini V, Mauri MC, et al. A single blind comparison of amisulpride, fluoxetine and clomipramine in the treatment of restricting anorectics. Prog Neuropsychopharmacol Biol Psychiatry. 2001; 25(5):1049-1059.

6. Halmi KA, Eckert E, LaDu TJ, Cohen J. Anorexia nervosa. Treatment efficacy of cyproheptadine and amitriptyline. Arch Gen Psychiatry. 1986; 43(2):177-181.

7. Biederman J, Herzog DB, Rivinus TM, et al. Amitriptyline in the treatment of anorexia nervosa: A double-blind, placebo-controlled study. J Clin Psychopharmacol. 1985;5(1):10-16.

8. Attia E, Haiman C, Walsh BT, Flater SR. Does fluoxetine augment the inpatient treatment of anorexia nervosa? Am J Psychiatry. 1998;155(4): 548-551.

9. Walsh BT, Kaplan AS, Attia E, et al. Fluoxetine after weight restoration in anorexia nervosa: A randomized controlled trial. JAMA. 2006; 295(22):2605-2612.

10. Kaye WH, Nagata T, Weltzin TE, et al. Double-blind placebo-controlled administration of fluoxetine in restricting- and restricting-purging-type anorexia nervosa. Biol Psychiatry. 2001;49(7):644-652.

11. Fassino S, Leombruni P, Daga G, et al. Efficacy of citalopram in anorexia nervosa: A pilot study. Eur Neuropsychopharmacol. 2002; 12(5):453-459.

12. Vandereycken W, Pierloot R. Pimozide combined with behavior therapy in the short-term treatment of anorexia nervosa. A doubleblind placebo-controlled cross-over study. Acta Psychiatr Scand. 1982;66(6):445-450.

13. Vandereycken W. Neuroleptics in the short-term treatment of anorexia nervosa. A double-blind placebo-controlled study with sulpiride. Br J Psychiatry. 1984;144:288-292.

14. Mondraty N, Birmingham CL, Touyz S, Sundakov V, Chapman L, Beumont P. Randomized controlled trial of olanzapine in the treatment of cognitions in anorexia nervosa. Australas Psychiatry. 2005; 13(1):72-75.

15. Brambilla F, Garcia CS, Fassino S, et al. Olanzapine therapy in anorexia nervosa: Psychobiological effects. Int Clin Psychopharmacol. 2007; 22(4):197-204.

16. Bissada H, Tasca GA, Barber AM, Bradwejn J. Olanzapine in the treatment of low body weight and obsessive thinking in women with anorexia nervosa: A randomized, double-blind, placebo-controlled trial. Am J Psychiatry. 2008;165(10):1281-1288.

17. Gross HA, Ebert MH, Faden VB, Goldberg SC, Nee LE, Kaye WH. A double-blind controlled trial of lithium carbonate primary anorexia nervosa. J Clin Psychopharmacol. 1981;1(6):376-381.

18. Szmukler GI, Young GP, Miller G, Lichtenstein M, Binns DS. A controlled trial of cisapride in anorexia nervosa. Int J Eat Disord. 1995; 17(4):347-357.

19. Kaplan AS, Howlett A. Pharmacotherapy for anorexia nervosa. In: Grilo CM, Mitchell JE, editors. The Treatment of Eating Disorders: A Clinical Handbook. New York, NY: Guilford Press; 2010.

20. Garner DM, Vitousek KM, Pike KM. Cognitive-behavioral therapy for anorexia nervosa. In: Garner DM, Garfinkel PE, editors. Handbook of Treatment for Eating Disorders. New York, NY: Guilford Press; 1997:94-144. 
21. Channon S, de Silva P, Hemsley D, Perkins R. A controlled trial of cognitive-behavioural and behavioural treatment of anorexia nervosa. Behav Res Ther. 1989;27(5):529-535.

22. Serfaty MA, Turkington D, Heap M, Ledsham L, Jolley E. Cognitive therapy versus dietary counselling in the outpatient treatment of anorexia nervosa: Effects of the treatment phase. Eur Eat Disord Rev. 1999;7(5).334-350.

23. Ball J, Mitchell P. A randomized controlled study of cognitive behavior therapy and behavioral family therapy for anorexia nervosa patients. Eat Disord. 2004;12:303-314.

24. McIntosh VV, Jordan J, Carter FA, et al. Three psychotherapies for anorexia nervosa: A randomized, controlled trial. Am J Psychiatry. 2005;162(4):741-747.

25. Pike KM, Walsh BT, Vitousek K, Wilson GT, Bauer J. Cognitive behavior therapy in the posthospitalization treatment of anorexia nervosa. Am J Psychiatry. 2003;160(11):2046-2049.

26. Bjorck C, Clinton D, Sohlberg S, Hallstrom T, Norring C. Interpersonal profiles in eating disorders: Ratings of SASB self-image. Psychol Psychother. 2003;76(Pt 4):337-349.

27. Fairburn CG, Norman PA, Welch SL, O'Connor ME, Doll HA, Peveler RC. A prospective study of outcome in bulimia nervosa and the long-term effects of three psychological treatments. Arch Gen Psychiatry. 1995;52(4):304-312.

28. Russell GF, Szmukler GI, Dare C, Eisler I. An evaluation of family therapy in anorexia nervosa and bulimia nervosa. Arch Gen Psychiatry. 1987;44(12):1047-1056.

29. Morgan HG, Russell GF. Value of family background and clinical features as predictors of long-term outcome in anorexia nervosa: Four-year follow-up study of 41 patients. Psychol Med. 1975;5(4):355-371.

30. Hall A, Crisp AH. Brief psychotherapy in the treatment of anorexia nervosa. Outcome at one year. $\mathrm{Br} J$ Psychiatry. 1987;151:185-191.

31. Robin AL, Siegel PT, Moye AW, Gilroy M, Dennis AB, Sikand A. A controlled comparison of family versus individual therapy for adolescents with anorexia nervosa. J Am Acad Child Adolesc Psychiatry. 1999;38(12):1482-1489.

32. le Grange D, Eisler I, Dare C, Russell GFM. Evaluation of family treatments in adolescent anorexia nervosa: A pilot study. Int J Eat Disord. 1992;12(4):347-357.

33. Eisler I, Dare C, Hodes M, Russell G, Dodge E, le Grange D. Family therapy for adolescent anorexia nervosa: The results of a controlled comparison of two family interventions. J Child Psychol Psychiatry. 2000;41(6):727-736.

34. Eisler I, Simic M, Russell GF, Dare C. A randomised controlled treatment trial of two forms of family therapy in adolescent anorexia nervosa: A five-year follow-up. J Child Psychol Psychiatry. 2007;48(6): $552-560$.

35. Geist R, Heinmaa M, Stephens D, Davis R, Katzman DK. Comparison of family therapy and family group psychoeducation in adolescents with anorexia nervosa. Can J Psychiatry. 2000;45(2):173-178.

36. Lock J, Agras WS, Bryson S, Kraemer HC. A comparison of short- and long-term family therapy for adolescent anorexia nervosa. J Am Acad Child Adolesc Psychiatry. 2005;44(7):632-639.

37. Dare C, Eisler I, Russell G, Treasure J, Dodge L. Psychological therapies for adults with anorexia nervosa: Randomised controlled trial of outpatient treatments. Br J Psychiatry. 2001;178:216-221.

38. Bachar E, Latzer Y, Kreitler S, Berry EM. Empirical comparison of two psychological therapies. Self psychology and cognitive orientation in the treatment of anorexia and bulimia. J Psychother Pract Res. 1999; $8(2): 115-128$.

39. Treasure J, Todd G, Brolly M, Tiller J, Nehmed A, Denman F. A pilot study of a randomised trial of cognitive analytical therapy vs educational behavioral therapy for adult anorexia nervosa. Behav Res Ther. 1995; 33(4):363-367.

40. Crisp AH, Norton K, Gowers S, et al. A controlled study of the effect of therapies aimed at adolescent and family psychopathology in anorexia nervosa. Br J Psychiatry. 1991;159:325-333.
41. Gowers SG, Clark A, Roberts C, et al. Clinical effectiveness of treatments for anorexia nervosa in adolescents: Randomised controlled trial. Br J Psychiatry. 2007;191:427-435.

42. Olmsted MP, McFarlane TL, Carter JC, Trottier K, Woodside DB, Dimitropoulos G. Inpatient and day hospital treatment for anorexia nervosa. In: Grilo CM, Mitchell JE, editors. The Treatment of Eating Disorders: A Clinical Handbook. New York, NY: Guilford Press; 2010.

43. Mehler PS, Birmingham CL, Crow SJ, Jahraus JP. Medical complications of eating disorders. In: Grilo $\mathrm{CM}$, Mitchell JE, editors. The Treatment of Eating Disorders: A Clinical Handbook. New York, NY: Guilford Press; 2010.

44. Keel PK, Haedt A. Evidence-based psychosocial treatments for eating problems and eating disorders. J Clin Child Adolesc Psychol. 2008; 37(1):39-61.

45. Halmi KA, Agras WS, Crow S, et al. Predictors of treatment acceptance and completion in anorexia nervosa: Implications for future study designs. Arch Gen Psychiatry. 2005;62(7):776-781.

46. APA Task Force on Promotion and Dissemination of Psychological Procedures, Division of Clinical Psychology. Training and dissemination of empirically-validated psychological treatments: Reports and recommendations. Clin Psychol. 1995;48:3-23.

47. National Institute of Clinical Excellence. Eating disorders: Core interventions in the treatment and management of anorexia nervosa, bulimia nervosa, and related eating disorders. London: National Institute for Clinical Excellence; 2004

48. Bulik CM. UCAN: Uniting couples (in the treatment of) anorexia nervosa. University of North Carolina Chapel Hill. In: NIH Research Portfolio Online Reporting [Internet]. Bethesda, MD: National Institutes of Health (US). Available at: http://projectreporter.nih.gov/project_info_description.cfm?aid=7666092\& icde=5064637 NIMH Identifier: 5R01MH082732-03. Accessed 2010 Aug 27.

49. Lock JD. Cognitive remediation therapy for anorexia nervosa. In: NIH Research Portfolio Online Reporting [Internet]. Bethesda, MD: National Institutes of Health (US). Available at: http://projectreporter.nih.gov/ project_info_description.cfm?aid=7664954\&icde=5064637 NIMH Identifier: 5R01MH082706-03. Accessed 2010 Aug 27.

50. Marcus MD. Emotional acceptance behavior therapy for anorexia nervosa. In: NIH Research Portfolio Online Reporting [Internet]. Bethesda, MD: National Institutes of Health (US). Available at: http:// projectreporter.nih.gov/project_info_description.cfm?aid=7664526\& icde=5064637 NIMH Identifier: 5R01MH082685-03. Accessed 2010 Aug 27.

51. Steinglass JE. Addressing fear of food in anorexia nervosa. In: NIH Research Portfolio Online Reporting [Internet]. Bethesda, MD: National Institutes of Health (US). Available at: http://projectreporter.nih.gov/project_info_description.cfm?aid=7682999 \&icde $=5064637$ NIMH Identifier: 5R01MH082736-03. Accessed 2010 Aug 27.

52. Kaye WH, Bulik CM, Thornton L, Barbarich N, Masters K. Comorbidity of anxiety disorders with anorexia and bulimia nervosa. Am J Psychiatry. 2004;161(12):2215-2221.

53. Keel PK, Klump KL, Miller KB, McGue M, Iacono WG. Shared transmission of eating disorders and anxiety disorders. Int J Eat Disord. 2005;38(2):99-105.

54. Attia E. Olanzapine versus placebo for outpatients with anorexia nervosa. In: Clinicaltrials.gov [Internet]. Bethesda, MD: National Institutes of Health (US). Available at: http://clinicaltrials.gov/ show/NCT01170117 NLM Identifier: NCT01170117. Accessed 2010 Aug 27.

55. Zipfel S. Focal psychodynamic psychotherapy, cognitive-behavioral therapy and treatment as usual in outpatients with anorexia nervosa. In: International Standard Randomised Controlled Trial Number Register [Internet]. Germany. Available at: http://www.controlled-trials.com/isrctn/ pf/72809357 ISRCTN Identifier: ISRCTN72809357. Accessed 2010 Aug 27. 
56. Hay P. A randomized controlled trial of the Loughborough eating disorders activity therapy versus general cognitive behavior therapy efficacy in reducing pathological exercise behaviors and cognitions. In: Australia New Zealand Clinical Trials Registry [Internet]. Australia. Avaliable at: http://www.anzctr.org.au/ACTRN12610000585022.aspx ACTRN Identifier: ACTRN12610000585022. Accessed 2010 Aug 27.

57. Lock J. Effectiveness of family-based versus individual psychotherapy in treating adolescents with anorexia nervosa. In: Clinicaltrials.gov [Internet]. Bethesda, MD: National Institutes of Health (US). Available at: http://clinicaltrials.gov/show/NCT00149786 NLM Identifier: NCT00149786. Accessed 2010 Aug 27.

58. Agras WS. Comparison of two types of family therapy in the treatment of adolescent anorexia nervosa. In: Clinicaltrials.gov [Internet]. Bethesda, MD: National Institutes of Health (US). Available at: http:// clinicaltrials.gov/show/NCT00610753 NLM Identifier: NCT00610753. Accessed 2010 Aug 27.

59. le Grange D. Comparing the effectiveness of three types of therapy for the treatment of anorexia nervosa in adolescents. In: Clinicaltrials.gov [Internet]. Bethesda, MD: National Institutes of Health (US). Available at: http://clinicaltrials.gov/show/NCT00183586 NLM Identifier: NCT00183586. Accessed 2010 Aug 27.

60. Schmidt U. A randomized controlled trial of the Maudsley model of treatment for adults with anorexia nervosa (MANTRA) compared to specialist supportive clinical management (SSCM) in outpatients with anorexia nervosa or eating disorder not otherwise specified (ED-NOS). In: International Standard Randomised Controlled Trial Number Register [Internet]. United Kingdom. Available at: http://isrctn.org/ISRCTN67720902 ISRCTN Identifier: ISRCTN67720902. Accessed 2010 Aug 27.
61. Eisler I. A multicentre randomised trial of the outcome, acceptability and cost-effectiveness of family therapy and multi-family day treatment compared with inpatient care and outpatient family therapy for adolescent anorexia nervosa. In: International Standard Randomised Controlled Trial Number Register [Internet]. United Kingdom. Available at: http://isrctn.org/ISRCTN11275465 ISRCTN Identifier: ISRCTN11275465. Accessed 2010 Aug 27.

62. Herpertz-Dahlmann B. Treatment of childhood and adolescent anorexia nervosa: day treatment versus inpatient treatment. In: International Standard Randomised Controlled Trial Number Register [Internet]. Germany. Available at: http://isrctn.org/ISRCTN67783402 ISRCTN Identifier: ISRCTN67783402. Accessed 2010 Aug 27.

63. Fichter M. Does internet based prevention reduce the risk of relapse in anorexia nervosa patients. In: International Standard Randomised Controlled Trial Number Register [Internet]. Germany. Available at: http://isrctn.org/ISRCTN20173615 ISRCTN Identifier: ISRCTN20173615. Accessed 2010 Aug 27.

64. Barbarich NC, McConaha CW, Halmi KA, et al. Use of nutritional supplements to increase efficacy of fluoxetine in the treatment of anorexia nervosa. Int J Eat Disord. 2004;35(1):10-15.

65. Feighner JP, Robins E, Guze SB, Woodruff RA, Winokur G, Munoz R. Diagnostic criteria for use in psychiatric research. Arch Gen Psychiat. 1972;26:57-63.

66. Russell GFM. Anorexia nervosa and bulimia nervosa. In: Russell GFM, Herson LA, editors. Handbook of psychiatry 4: The Neuroses and personality Disorders. Cambridge: Cambridge university press; 1983.
Psychology Research and Behavior Management

\section{Publish your work in this journal}

Psychology Research and Behavior Management is an international, peerreviewed, open access journal focusing on the science of psychology and its application in behavior management to develop improved outcomes in the clinical, educational, sports and business arenas. Specific topics covered include: Neuroscience, memory \& decision making; Behavior

\section{Dovepress}

modification \& management; Clinical applications; Business \& sports performance management; Social and developmental studies; Animal studies. The manuscript management system is completely online and includes a quick and fair peer-review system. Visit http://www.dovepress. com/testimonials.php to read real quotes from published authors. 